\title{
Three-dimensional eye position signals shape both peripersonal space and arm movement activity in the medial posterior parietal cortex
}

\author{
K. Hadjidimitrakis, R. Breveglieri, A. Bosco and P. Fattori*
}

Department of Human and General Physiology, University of Bologna, Bologna, Italy

Edited by:

Christos Constantinidis, Wake

Forest University, USA

Reviewed by:

Kae Nakamura, Kansai Medical

University, Japan

He Cui, Georgia Health Sciences

University, USA

\section{*Correspondence:}

P. Fattori, Department of Human and

General Physiology, University of

Bologna, Piazza di Porta San Donato

2, Bologna 40126, Italy

e-mail: patrizia.fattori@unibo.it
Research conducted over the last decades has established that the medial part of posterior parietal cortex (PPC) is crucial for controlling visually guided actions in human and non-human primates. Within this cortical sector there is area V6A, a crucial node of the parietofrontal network involved in arm movement control in both monkeys and humans. However, the encoding of action-in-depth by V6A cells had been not studied till recently. Recent neurophysiological studies show the existence in V6A neurons of signals related to the distance of targets from the eyes. These signals are integrated, often at the level of single cells, with information about the direction of gaze, thus encoding spatial location in 3D space. Moreover, 3D eye position signals seem to be further exploited at two additional levels of neural processing: (a) in determining whether targets are located in the peripersonal space or not, and (b) in shaping the spatial tuning of arm movement related activity toward reachable targets. These findings are in line with studies in putative homolog regions in humans and together point to a role of medial PPC in encoding both the vergence angle of the eyes and peripersonal space. Besides its role in spatial encoding also in depth, several findings demonstrate the involvement of this cortical sector in non-spatial processes.

Keywords: fixation depth, vergence, version, gaze, sensorimotor transformation, reaching, eye-hand coordination

\section{INTRODUCTION}

The posterior parietal cortex (PPC) of primates has been shown to be important for multisensory space representation and the control of goal directed action (Culham et al., 2006; Husain and Nachev, 2007). Traditionally, PPC has been considered a key node of the dorsal visual stream involved in the "vision for action" neural processing (Goodale and Milner, 1992). The PPC comprises the superior parietal lobule (SPL) and the inferior parietal lobule (IPL), separated by the intraparietal sulcus (IPS). The SPL of macaque monkeys encompasses several areas, as shown in Figure 1A. These regions include V6 and V6A in the anterior bank of the parieto-occipital sulcus (Galletti et al., 1999), the medial intraparietal area (MIP) in the medial bank of the IPS, [MIP, together with the most lateral part of V6A is included within the functionally defined parietal reach region (PRR, Snyder et al., 1997)], areas PE (area 5) and PE caudal (PEc), and PGm in the medial part of SPL (Pandya and Seltzer, 1982; Shipp et al., 1998; Bakola et al., 2010). The ventral intraparietal area (VIP), located at the fundus of IPS between SPL and IPL, can also be grouped with the SPL regions due to its functional properties (Colby et al., 1993).

The above areas form together an important network that performs the integration of visual and somatic spatial information necessary for the control of arm movements in space (Snyder et al., 1997; Buneo et al., 2002; Galletti et al., 2003; Breveglieri et al., 2006; McGuire and Sabes, 2011). Figure 1B summarizes the flow of information in SPL by illustrating the major connections of area V6A. This brain region receives visual information from the extrastriate areas V2, V3, V4, V6, and MST (Gamberini et al., 2009; Passarelli et al., 2011). Moreover, it receives input from areas MIP and VIP, where neurons with both visual and somatosensory sensitivity have been found (Colby and Duhamel, 1991). Additional somatosensory input may be relayed to V6A through PGm. V6A also shows strong reciprocal connections with LIP and area PG of the IPL, involved in encoding spatial parameters for ocular and manual actions (Barash et al., 1991; Heider et al., 2010). V6A, together with MIP and PEc, send a strong projection to the arm region of area F2 in the premotor cortex (Godschalk et al., 1995; Matelli et al., 1998; Raos et al., 2003). In summary, SPL areas through their reciprocal interactions, collectively process visual, somatosensory and motor information to program and control reaching movements.

The anatomical and neurophysiological evidence from monkeys is in line with data from neurological patients. Lesions in human SPL have been reported to produce deficits in the perception of the spatial relationship between objects and the subjects' own body that is manifested with inaccurate reaching movements (Critchley, 1953; Perenin and Vighetto, 1988; Wolpert et al., 1998). Furthermore, damages to SPL were shown to compromise more severely the depth component of visually guided arm movements (Baylis and Baylis, 2001; Danckert et al., 2009). The encoding by SPL of the visually guided behavior in depth has been addressed by relatively few studies (Lacquaniti et al., 1995; Bhattacharyya et al., 2009; Ferraina et al., 2009). In the PRR of 


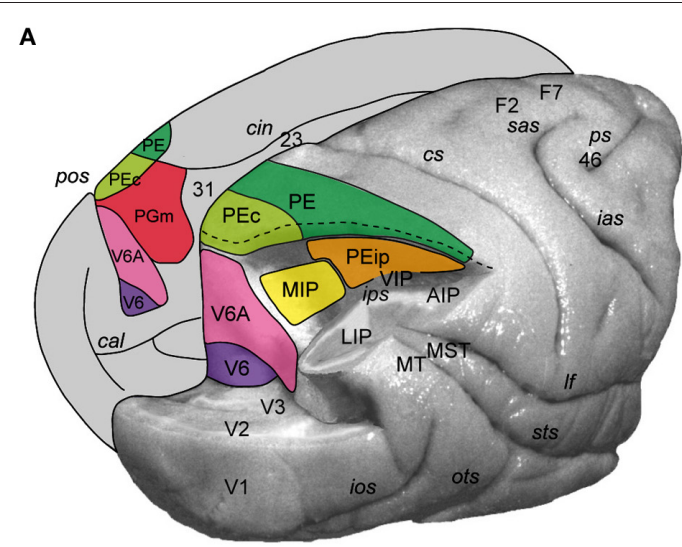

B

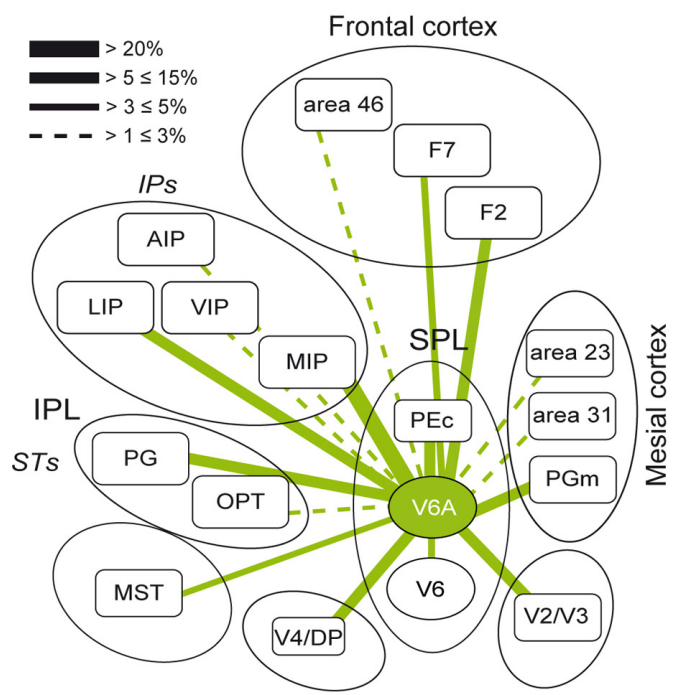

FIGURE 1 | (A) Location and extent of the areas that form the superior parietal cortex of the macaque brain. Posterolateral view of a partially dissected macaque brain modified from Galletti et al. (1996). The occipital pole has been partially removed and inferior parietal lobule of the right hemisphere has been cut away at the level of the fundus of the intraparietal sulcus to show the cortex of the medial bank of this sulcus. The occipital lobe of the same hemisphere has been cut away at the level of the fundus of the parieto-occipital and lunate sulci to show the cortex of the anterior bank of the parieto-occipital sulcus. The medial surface of the left hemisphere is drawn to show the location on it of all areas that extend medially. pos, parieto-occipital sulcus; cal, calcarine sulcus; cin, cingulate sulcus; ips, intraparietal sulcus; ios, inferior-occipital sulcus; ots, occipitotemporal sulcus; sts, superior temporal sulcus; If, lateral fissure; cs, central sulcus; sas, superior arcuate sulcus; ias, inferior arcuate sulcus; ps, principal sulcus; V6, area V6; V3, area V3; V2, area V2; V1, area V1; PEc, area caudal PE; PE, area PE; PEip, intraparietal area PE; MIP, medial intraparietal area, PGm, area medial PG; VIP, ventral intraparietal area; LIP, lateral intraparietal area; AIP, anterior intraparietal area; MT, middle temporal area; MST, medial superior temporal area; Brodmann's areas 23, 31, 46, and dorsal premotor areas F2 and F7 in are also shown. (B) Flow chart of the connections of V6A modified from Passarelli et al. (2011). Rostral/caudal brain areas are shown at the top/bottom part of the figure. The thickness of the lines is proportional to the strength of each connection. Areas in the ventral part of the parieto-occipital sulcus (V6, ventral V6A) are dominated by visual input, whereas as one proceeds toward the dorsal part of V6A sensory association and visuomotor/premotor connections prevail. Inferior parietal lobule areas Opt and PG and occipital areas V4/DP are also shown. monkeys, a strong influence of vergence angle on the activity of neurons involved in the planning of reaches has been demonstrated (Bhattacharyya et al., 2009), whereas in area PE signals related to the position of the hand prevailed (Ferraina et al., 2009).

Eye position signals are critical in the visuomotor transformations performed by the PPC, as they are used to compute the position of visual targets with respect to the body (Andersen and Mountcastle, 1983; Andersen et al., 1990; Bremmer et al., 1997). The computation of target location in an egocentric frame of reference is realized through the modulation of a visual response by gaze position in what has been referred to as a "gain field" mechanism (Zipser and Andersen, 1988). Gain fields have been demonstrated in many areas of both the dorsal and ventral stream, in the primary visual cortex and in subcortical structures as well (Salinas and Sejnowski, 2001). Regarding the encoding of target location in depth, a model of disparity-selective neurons gain modulated by the vergence angle has been proposed (Pouget and Sejnowski, 1994) and subsequent studies in PRR and lateral intraparietal area (LIP) provided experimental support for it (Genovesio and Ferraina, 2004; Bhattacharyya et al., 2009). Apart from the spatial localization of targets, gain modulations of activity by eye position have been linked with systematic biases in space representation that serve behaviorally important perceptual and motor actions. For example, in the primary visual cortex the majority of neurons that represent the peripheral visual field increase their firing as the eyes attain eccentric fixation, thus facilitating the processing of targets presented at straight-ahead directions (Durand et al., 2010). Similarly, in areas V2 and V4 most of the neurons with distance tuning for near space preferred also downward gaze positions (Rosenbluth and Allman, 2002) and this could reflect the fact that it is more usual to look down when we fixate near targets. These associations between eye position signals and behavioral context could be the result of learning and adaptation processes.

In the rest of this review we focus on the multiple functions of 3D eye position signals in SPL, in particular in area V6A. We review recent neurophysiological studies performed by our group to address whether signals related to vergence angle and to the encoding of the peripersonal space are processed in area V6A. We also present the results of studies in putative homologue regions in human SPL that also addressed these two issues. Furthermore, we will review evidence showing the influence of eye position activity on the spatial tuning of reaching discharges. In addition, we will review recent findings from human studies that support the involvement of SPL in non-spatial cognitive processes.

\section{CODING OF TARGET POSITION IN 3D SPACE: INTEGRATION OF VERGENCE AND VERSION SIGNALS IN V6A}

To estimate the position of a foveated target in 3D space, information about the direction of gaze (version) and the depth of fixation (vergence) is needed. Electrophysiological studies in various PPC areas found that neuronal activity was modulated by version signals (Andersen et al., 1990; Galletti et al., 1995; Bremmer et al., 1997; Nakamura et al., 1999). Similarly, PPC neurons affected by vergence signals were reported (Genovesio and Ferraina, 2004). In addition, vergence is a valid cue for distance estimation within the space that can be reached by the hands (Viguier et al., 2001). 
A combined encoding of the direction and depth of gaze in single cells has been only demonstrated in a small number of cells in area 7a of IPL (Sakata et al., 1980).

In our study (Breveglieri et al., 2012), we set out to investigate the effect of vergence angle and its interaction with version signals in V6A. Two monkeys were trained to fixate targets located within the peripersonal space at different distances and directions from the eyes (Figure 2A). In total, 74\% of the cells were affected by the vergence and/or the version angle, with the majority of the modulated cells being affected by both gaze variables. Figure $2 \mathbf{B}$ shows a neuron displaying its maximum discharge when the gaze was directed to the near and contralateral space. In addition, this neuron showed a linear increase of activity as the gaze shifted from far to near and from ipsilateral to contralateral positions. A multilinear regression analysis showed that in the majority of modulated neurons $(\sim 85 \%)$ vergence and version had a linear effect on the neural activity. This finding suggests that intermediate depths and straight-ahead gaze positions did not activate maximally most of V6A cells. When the average activity of all cells modulated by vergence (Figure 2C, left) and version (Figure 2C, right) was plotted as a population spike density function, the central space evoked significantly (permutation test, $p<0.05$ ) lower activity compared to contralateral and ipsilateral space (Figure 2C, right). At the same time, no difference was found in the average activity for near, intermediate and far positions (Figure 2C, left). While the underrepresentation of central gaze positions confirms studies of eye position encoding in two dimensional space (Galletti et al., 1995; Nakamura et al., 1999), the monotonic tuning of most V6A cells by vergence is a new finding that is in agreement with evidence from area LIP (Genovesio and Ferraina, 2004). At the population level, monotonic cells could equally increase their activity toward near and far peripersonal space $(n=38$, $44 \%$ near, $n=49,56 \%$ far). This result was in contrast with the study of Genovesio and Ferraina (2004), where the majority of cells increased their activity with increasing vergence. This suggests that there is a different encoding of depth in SPL compared to IPL. In neurons showing a linear effect of version there was a trend for representing contralateral space locations that was not statistically significant ( $\chi^{2}$ test, $p>0.05$ ). In summary, our study revealed a strong effect of signals related to vergence angle in area V6A. Furthermore, these signals were often integrated with version information in single neurons thus allowing the encoding of locations in $3 \mathrm{D}$ space.

The putative human homologue of monkey V6A is presumably located in the dorsal part of the parieto-occipital sulcus (POs), anterior to the human V6 (Pitzalis et al., 2006; CavinaPratesi et al., 2010). In that region, modulations of activity by the direction of gaze (Law et al., 1998; Williams and Smith, 2010), but also by eyes' vergence have been reported (Quinlan and Culham, 2007). In the latter study, the authors found higher activity in the dorsal POs while the subjects were viewing moving or stationary stimuli located in near space, compared to the intermediate or far space. It should be noted that the intermediate spatial location was within the limits of reachable space, whereas the far location was outside of reachable space. Fixating small LEDs placed at the same range of distances (near, intermediate and far) resulted in the same pattern of modulation in dPOS. Given the consistency
A

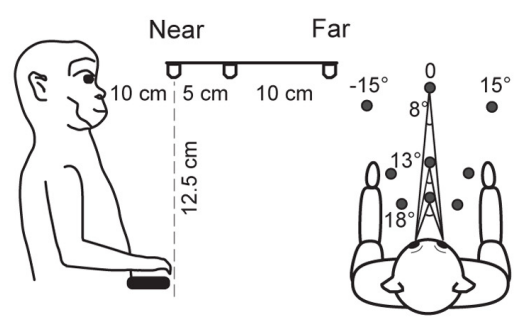

B
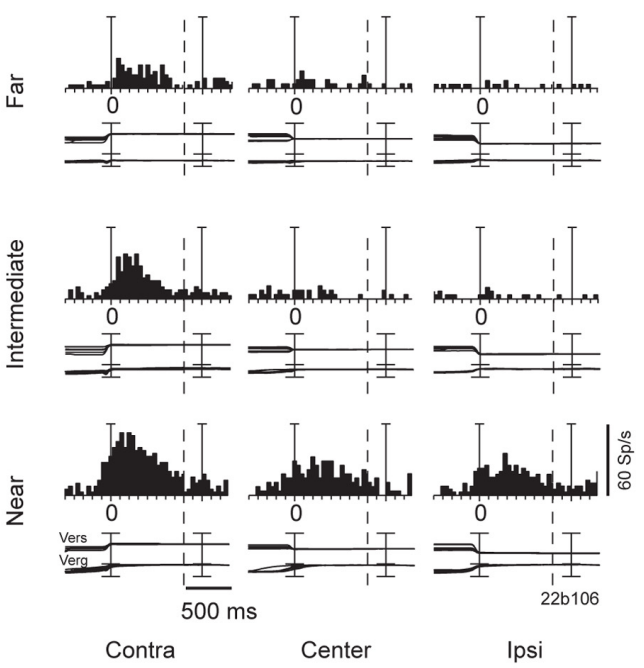

C
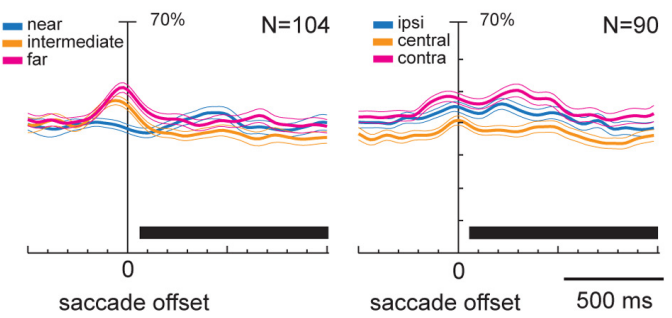

FIGURE 2 | (A) Scheme of the experimental setup set up used for the fixation-in-depth and reaching-in-depth tasks. Exact distances and angles of the targets are indicated in the lateral view (left) and top view (right), respectively. (B) Example of a V6A neuron modulated by both version and vergence during fixation. The discharge to the nine LEDs arranged from near (bottom) to far (top) was aligned twice (at the start of the fixation and at the LED change; dashed line: point where trials were cut because of double alignment). From left to right, the behavioral events are: LED onset, saccade offset (first alignment marker), LED change (second alignment marker). The cell has a clear preference for the near-contralateral space. Bin size for spike histograms: $20 \mathrm{~ms}$; scale for version and vergence traces is 100 and $20^{\circ}$, respectively. (C) Population activity of V6A neurons modulated during fixation. Population average spike density functions (SDF) were constructed by ranking the response for each tested row of fixation targets. The thick lines indicate average normalized SDF; the light lines indicate variability bands (SEM). The peak of the SDF of the row showing the maximum activity was set to $1(100 \%)$ and was used to normalize the SDF curves of the other rows. Activity is aligned with the offset of the saccade. The black rectangles below each plot indicate the periods where the permutation test was run. In the left plot, no statistical difference was observed between the curves (permutation test, $p>0.05$ ), whereas in the right, the central row is statistically different from the other two (permutation test, $p<0.05)$. Scale on abscissa: $100 \mathrm{~ms} /$ division; vertical scale: $70 \%$ of normalized activity (10\% per division). (A-C) panels were modified from Breveglieri et al. (2012). 
of their results, Quinlan and Culham (2007) attributed them to the vergence of the eyes. Their findings are in line with our neurophysiological data from monkey V6A and argue strongly for the encoding of $3 \mathrm{D}$ space by early visuomotor areas in POs, in both human and non-human primates.

A subsequent question we examined was the influence of depth information on the reaching activity. To this end, monkeys were trained to fixate first and then reach the targets of the same experimental set up where the fixation task was performed (Figure 2A). Figure 3 illustrates an example neuron recorded with the reaching-in-depth task. This neuron displayed a modulation by depth that started from movement planning and was present also during movement execution and holding of the target epochs. In all these epochs the neuron preferred far positions. In addition, during movement and target holding the cell showed a preference for central and ipsilateral targets. In most of the neurons recorded, there was an effect of both depth and direction signals in at least one motor epoch, a finding similar to the combined influence of vergence and version signals found in the fixation task (Breveglieri et al., 2012). Furthermore, the incidence and strength of depth modulations increased during the task progress. These findings suggest the presence of depth information in V6A not only for the purpose of target localization, but also for movement control (Hadjidimitrakis et al., 2011a).

Few studies have so far documented the effect of eye vergence signals in the cortex of non-human primates. Sakata et al. (1980) found in the IPL area PG-PFG cells that were modulated by both gaze direction and depth of fixation, while fewer cells were influenced by only one of these factors. Rosenbluth and Allman (2002) found that a significant number of neurons (30-50\%) in visual areas V1, V2, V4 were influenced by either gaze direction, depth of fixation, or their interaction, though they did not report the number of cells affected by both signals. These areas, directly connected to V6A (see also Figure 1B) may be sources of vergence information to area V6A, which receives also input from the posterior parietal areas LIP and PG (Gamberini et al., 2009), where vergence angle has been reported to have an effect on presaccadic and fixation activities, respectively (Sakata et al., 1980; Genovesio and Ferraina, 2004). Other possible sources of vergence input are the medial superior temporal area (MST) and the frontal eye fields (FEF). Both regions contain neurons with vergence-related activity (Inoue et al., 1998; Gamlin and Yoon, 2000; Akao et al., 2005) and send efferent inputs to V6A (Gamberini et al., 2009; Passarelli et al., 2011). In addition, modulations of neural activity by vergence have been found in the VIP (Colby et al., 1993) and in visual areas V2 and V4 (Dobbins et al., 1998; Rosenbluth and Allman, 2002), all of them directly connected with V6A, as also summarized in Figure 1B. The two extrastriate areas, in particular, send strong inputs to the ventral part of V6A (Passarelli et al., 2011), so are likely candidates to provide vergence signals to V6A.

\section{ENCODING OF PERIPERSONAL SPACE IN MONKEY AND HUMAN V6A}

The space within arm's reach is also termed peripersonal space (Previc, 1998). Several lines of evidence from monkey neurophysiology seem to support the view that the encoding of peripersonal space is being processed in PPC, and more in particular in SPL. For instance, neurons with multimodal (e.g., visual-tactile) receptive fields (RFs) have been reported in several PPC areas (Colby et al., 1993; Avillac et al., 2005). Overrepresentation of body

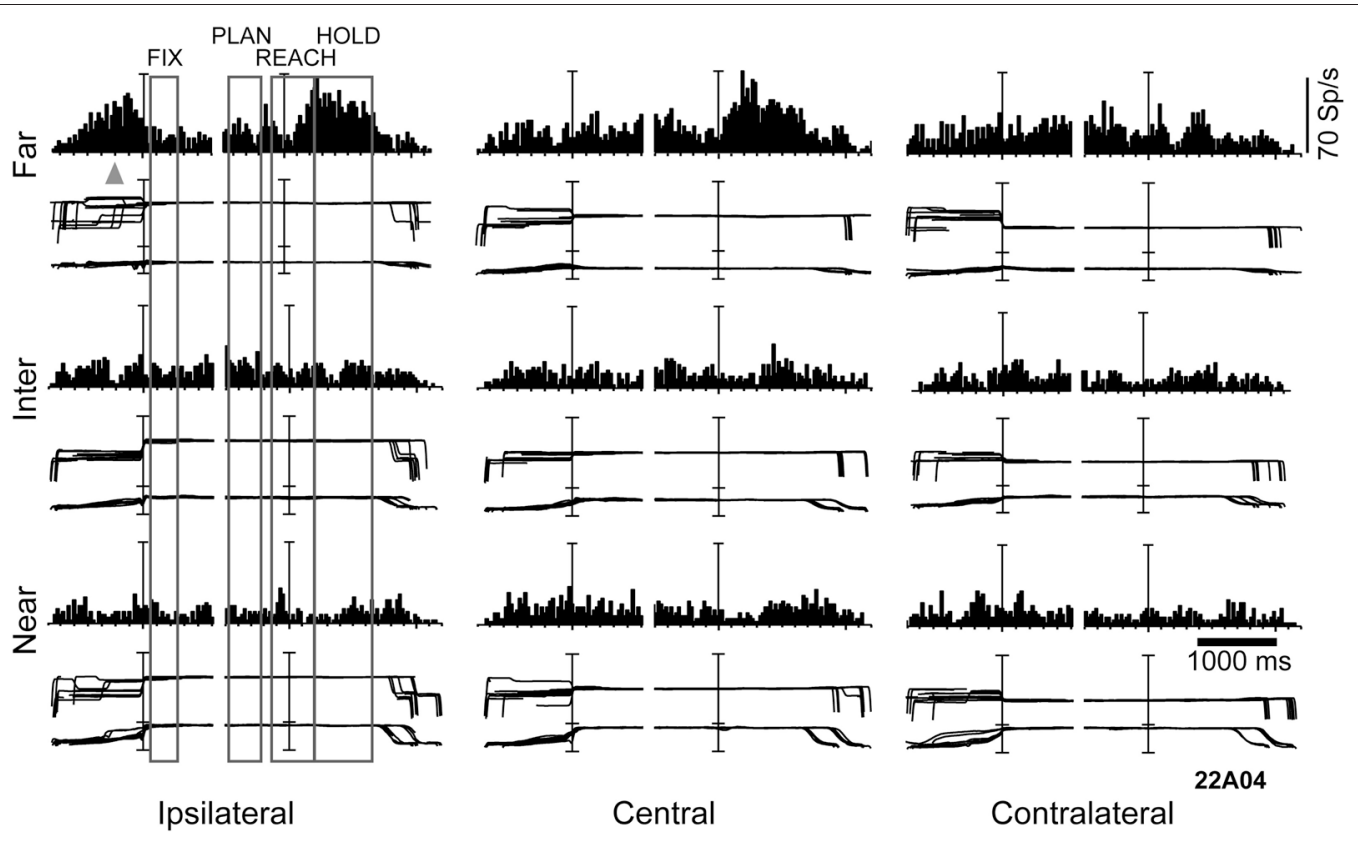

FIGURE 3 | Example of a V6A neuron modulated by depth and direction signals during several epochs of a reaching-in-depth task. Target LEDs were arranged as in Figure 2B. Spike histograms and eye traces were aligned twice, at the fixation onset and at the start of movement. This cell prefers far space during movement planning, execution and holding of the target epochs. In the last two epochs it was also tuned for ipsilateral space. The gray triangle indicates the mean time of LED onset. Bin size for spike histograms; $20 \mathrm{~ms}$; scale for version and vergence traces is 100 and $20^{\circ}$, respectively. 
parts (e.g., face or hand) (Colby et al., 1993; Breveglieri et al., 2002; Graziano and Cooke, 2006) that are behaviorally important has been found in several SPL areas and these representations show adaptation after training or are modified depending on the task performed (Graziano et al., 2000). In addition, representational biases in the encoding of visual space could be attributed to the natural position of the effectors in space. In this context, areas of the dorsal visual pathway show a preferential encoding of the lower visual field where the hands are usually located (Previc, 1998). Area V6A belongs to the dorsal pathway and the vast majority of V6A neurons have their visual RFs in the lower contralateral visual field (Galletti et al., 1996, 1999). In addition, many V6A neurons have somatosensory RFs centered on the arm (Breveglieri et al., 2002) and even more show spatially tuned responses during reaches in 3D space (Fattori et al., 2005). Recently, the orientation of the hand and the type of hand grip were found to affect the activity of V6A cells during reach-to-grasp movements (Fattori et al., 2009, 2010).

The hypothesis that SPL could process specific information related to the peripersonal space prompted us to study (Hadjidimitrakis et al., 2011b) whether there is a preferential encoding of that part of space compared to the extrapersonal space in V6A. To test this, we studied the oculomotor and fixation responses of V6A neurons to targets presented at near and far parts of space. We adopted an experimental configuration used in several human studies (Gallivan et al., 2009, 2011) with small LED targets arranged in a horizontal board below eye level, in order to simulate the natural perimanual space. The monkeys were trained to direct their gaze to the LED targets located in different positions in the 3D space without performing any arm movement (Figure 4A). Ten targets were divided into two rows, one located centrally, in the mid-sagittal plane, and the other located contralateral to the recording site, in a parasagittal plane (Figure 4A). In several neurons where isolation was held constant for longer periods, the horizontal board was translated so the targets were distributed between the central row and a parasagittal row in the ipsilateral space. Even though no reaches were performed by the animals, we checked that the two nearest LED targets in each row were within reaching distance $(<30 \mathrm{~cm})$.

Neural activity was quantified in a time epoch around the saccadic eye movement (perisaccadic activity) and during the subsequent fixation period (fixation activity). A One-Way ANOVA test performed independently in each row showed, on average, a significant effect $(P<0.05)$ of the depth of fixation in perisaccadic and fixation epochs in about 30 and $40 \%$ of V6A cells, respectively. About 15\% of the cells were modulated by the depth of fixation in both epochs. Figure 4B shows the firing activity of a neuron with a tonic pattern during fixation. The greatest activity was evoked while the eyes were fixed on the nearest target, irrespectively of gaze angle (Figure 4B). Activity consistently decreased when fixation was directed to targets located farther and farther. In addition, at constant fixation depth the responses showed an increasing trend from contralateral to ipsilateral space (Figure 4B). Thus, the neuron was significantly modulated by the depth of fixation and displayed monotonic tuning for near and ipsilateral space (ANOVA, $P<0.05$ ). We applied a Bonferroni post hoc test on the ANOVA significant cells to define the preferred position in depth in each part of space. Perisaccadic activity (Figure 4C, left), was typically higher for saccades to the two nearest targets: $60-75 \%$ of the cells preferred one of these LEDs in the different rows. We compared the incidence of preference for the two nearest (reachable) targets with the three that were outside the peripersonal, reachable space. The preference for reachable locations was highly significant $\left(\chi^{2}\right.$ test, two nearest LEDs against the three farthest ones, $P<0.0001)$. In neurons with modulation during fixation, there was also a strong preference for the peripersonal space (Figure 4C, right). Their percentage ranged from 65 to $74 \%$ depending on the different parts of space tested: 74\% (45/61) for straight-ahead, 73\% (56/77) for contralateral and 65\% (19/29) for ipsilateral locations. The preference for fixating targets placed within the reachable space with respect to those positioned outside was again highly significant $\left(\chi^{2}\right.$ test, two nearest LEDs against the three farthest ones, $P<0.0001)$. It was also demonstrated that the fixation distance of $45 \mathrm{~cm}$ (third LED in each row), which was outside the monkey's reachable space, was the least represented in our cell population (Figure 4C, right). This "gap zone" of fixation preference, between the reachable and unreachable space, could serve to signal targets that are definitely within or beyond reach. Overall, at the population level perisaccadic and fixation activity of V6A neurons showed a strong bias for representing the $3 \mathrm{D}$ space within reaching distance (Figure 4D).

Our study showed many similarities with a human functional imaging study performed by Gallivan et al. (2009). They used an experimental setup similar to ours with objects being placed in the perimanual space, below eye level. The authors reported that passive viewing of reachable objects evoked higher activity compared to objects located in non-reachable space in the superior part of parieto-occipital cortex (SPOC). The SPOC, which was also activated during arm reaching movements (CavinaPratesi et al., 2010), includes the cortex anterior to the human homologue of V6 (Pitzalis et al., 2006), i.e., the putative area V6A. In that study, the fixation point was kept constantly at far locations. Objects within reachable space evoked stronger activation in SPOC, and it was suggested that this modulation could be related to the objects' reachability. The authors proposed that a reachability signal could be extracted in SPOC by the combination of gaze and object depth signals. Given the response properties of cells modulated by depth described in our study (Hadjidimitrakis et al., 2011b), it is likely that both human SPOC and macaque V6A encode the difference in depth between current fixation and location of objects to be grasped. In this context, the abundance of strong tuning for near space observed could be a result of an adaptive cognitive mechanism that satisfies two conditions: the natural tendency for fixating far and the necessity to respond to behaviorally relevant stimuli appearing in the near space. Beyond this hypothesis, both our study (Hadjidimitrakis et al., 2011b), and that of Gallivan et al. (2009) suggest that in V6A visual, eye position and motor related information is integrated to encode more cognitive variables such as motor affordance and/or potential actions (Andersen and Cui, 2009; Cisek and Kalaska, 2010; Macaluso and Maravita, 
A
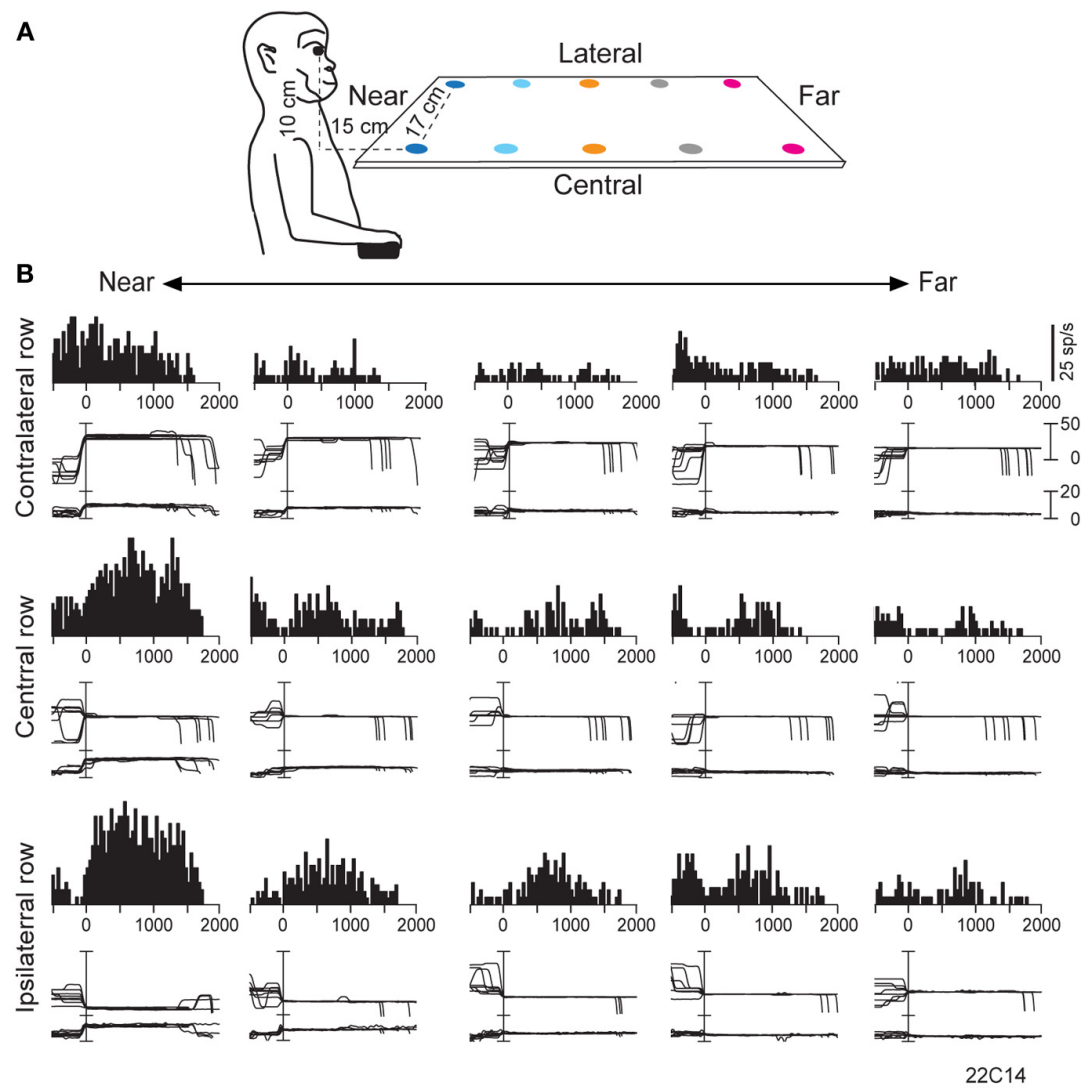

C
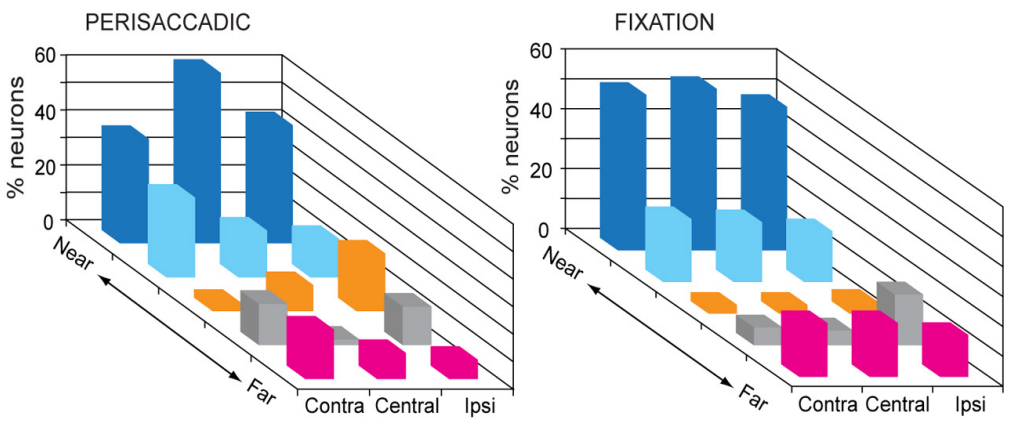

D
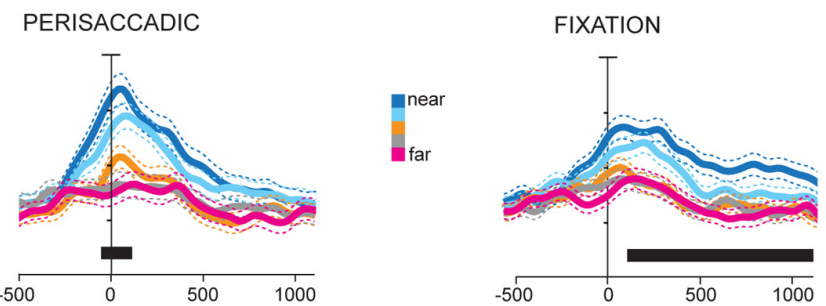

FIGURE 4 | (A) Scheme of the set up used for the fixation-in-depth task below eye level. The monkey was fixating in darkness one of the LEDs embedded in each panel. The LEDs are depicted with different colors according to their distance from a frontal plane passing from monkey's eyes. (B) Example of a V6A neuron modulated by vergence during fixation below eye level. Top/Middle/Bottom: neural responses (peristimulus time histograms) and eye traces to the five LEDs of the contralateral/central/ipsilateral space arranged from near (left) to far (right), aligned at the end of the saccade. This cell prefers targets located at near and ipsilateral space. Other conventions as in Figure 3. (C) Frequency histogram of the positions that neurons preferred in perisaccadic $(N=91)$ and fixation $(N=167)$ epochs. Ipsi and Contra indicate fixation position with respect to the recording hemisphere. Center refers to the straight ahead of the monkey. In both epochs there is a clear preference for near, reachable targets across all space. (D) Population activity for each target position, illustrated with a different color, of V6A neurons with activity modulated during perisaccadic (left) and fixation (right) epochs. Activity is aligned on the saccade onset in both panels. Other conventions as in Figure 2C. (A-D): Adapted from Hadjidimitrakis et al. (2011b). 
2010). In line with this, there is evidence from a subsequent study by Gallivan et al. (2011) that activation in SPOC region was found to be correlated with the hand dominance, with the later being crucial in defining the subjects' typical reachable space.

\section{CONGRUENCE OF THE SPATIAL TUNING BETWEEN FIXATION- AND REACHING-RELATED ACTIVITY}

As described in the previous section, neural discharges during the presentation and ocular fixation of targets can be an important cue to estimate whether they are reachable or not. But what happens when an arm reaching movement is actually performed? Does the activity during arm movement show the same spatial tuning with that of the visual fixation-related discharges? It has been suggested that PPC neurons integrate spatially consistent retinal, eye and hand information into a "global tuning field, GTF" (Mascaro et al., 2003). This type of common tuning of different effectors could be advantageous for the control of eye-hand coordination. According to Caminiti and colleagues, optic ataxia could be the result of the breakdown of GTF in SPL (BattagliaMayer and Caminiti, 2002). Evidence for neurons with consistent spatial tuning between eye position, arm movement and position signals has been reported in the SPL (Battaglia-Mayer et al., 2001). In that study, in a center-out reaching task about $60 \%$ of the neurons showed GTF in several epochs and across various oculomanual tasks. The same group found in area 7A a much lower incidence ( $\sim 25 \%$ ) of cells with GTF (Battaglia-Mayer et al., 2005).

We addressed this issue in another study of our group (Fattori et al., 2005), where monkeys were trained to perform 3D reaching movements toward foveated LED targets arranged on a plane (Figure 5A). The monkeys were required to fixate the targets and wait for a go cue signal (LED color change) to perform a body-out reach toward the LED and hold it for a variable time, till another cue (switching off of the LED) signaled the monkey to return to the initial hand position. Many neurons in V6A were spatially tuned (One-Way ANOVA, $P<0.05$ ), both during the outward movement execution (Figure 5B, Raw M1) and in the holding of target period (Figure 5B, Raw HOLD). Modulations in these two periods often coincided and in many cases they were spatially consistent. Figure 5C shows an example of this pattern of activity in a neuron that fired strongly not only when the hand moved, but also when it held targets located on the right. In addition, this neuron showed a preference for right space in the fixation period, i.e., it displayed a pattern of activity similar to the cells with GTF. To test whether the tuning of arm-related activity could be explained by gain modulation by eye position, fixation activity was substracted from the discharges in movement and hold epochs. An ANOVA $(P<0.05)$ then was performed to detect significant effects of target position on the residual firing activity. The substraction of fixation activity abolished the spatial tuning in about one-third of V6A neurons in both movement and holding periods (Figure 5B, Proper M1, Proper HOLD), whereas about $40 \%$ of the V6A maintained their spatial tuning. In line with our data are those obtained in area 7A in IPL (Heider et al., 2010). The authors, using a body-out reaching task very similar to ours, found that about $60 \%$ of the neurons changed their directional tuning from eye fixation till preparation and movement execution (Heider et al., 2010). Both their results and ours suggest that "global tuning" is probably implemented in PPC, but in a much more limited extent with respect to the one originally reported (Battaglia-Mayer et al., 2001).

Regardless of its incidence in the PPC, the concept of GTF has some interesting cognitive aspects. In everyday life and under certain conditions, when we want to reach and/or grasp an object, the eyes fixate on the target first and then the hand starts to move (Land et al., 1999; Hayhoe et al., 2003). In this context, the common spatial preference for eye and arm could be the result of a learned association between eye position and arm movement signals. Learning could be the result of many repetitions of combinations of eye and arm positions in space. The spatial coincidence would reinforce the response of neurons that are tuned (Ahissar et al., 1992; Rosenbluth and Allman, 2002). As a result, in these neurons, whenever the eyes move to a certain location, a plan that would carry the arm in the same location is formed. In this way, eye position signals can predict the sensory consequences of future motor actions (Land and Furneaux, 1997).

\section{NON-SPATIAL PROCESSING IN HUMAN SPL}

Apart from its crucial role in spatial perception and visually guided motor action, experimental evidence accumulated over the past decade suggests that PPC is also involved in nonspatial, higher order cognitive functions. Such functions include attention, categorization, reward, working memory, encoding of task rules, and behavioral context (for references see Culham and Kanwisher, 2001; Husain and Nachev, 2007; Gottlieb and Snyder, 2010). Most studies of cognitive processes in monkeys and humans have found the neural substrate of these functions to be located in areas of the IPL and this created a view of a gradient of increasing non-spatial, cognitive processing going from SPL to IPL (Husain and Nachev, 2007). Here, without claiming the opposite to this view, we focus on evidence that relates the SPL activity to cognitive processing.

Yantis and colleagues identified a region in the medial SPL of humans that was transiently activated during shifts of spatial attention (Yantis et al., 2002). In that study, subjects were presented with two stimulus streams of numerical cues at opposite peripheral locations (left-right). Their task was to attend one of the two streams for the presentation of a cue that signaled them, either to shift their attention to the unattended stream, or to maintain it on the attended one. The authors found that a region in the medial SPL showed a transient activation during the shift of attention, irrespectively of the direction of the shift. This area is likely homologous to macaque V6A, where modulations during covert shifts of attention have been reported (Galletti et al., 2010). Furthermore, in subsequent studies, the same human medial SPL region was found to exhibit transient activity in a variety of conditions: during non-spatial shifts of attention between objects, features and working memory representations, and during shifts between visual features that represented different categorization rules (Chiu and Yantis, 2009; Esterman et al., 2009). These findings suggest that SPL activity is related to the encoding of the change in task demands. In line with this assumption are the results of human studies performed by other groups. 
A

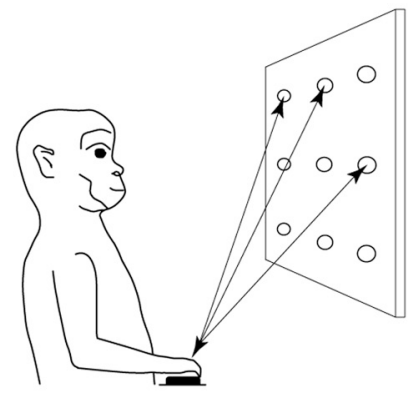

B

$\%$

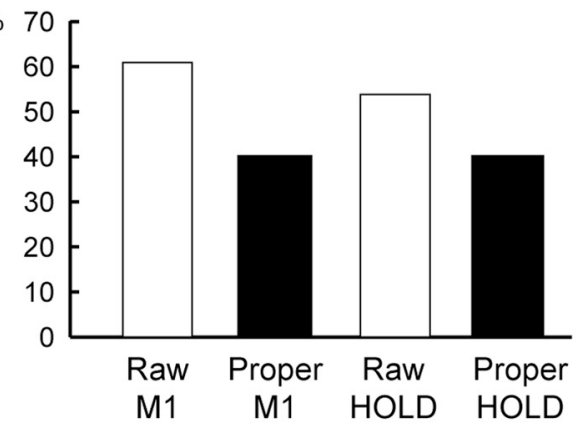

C

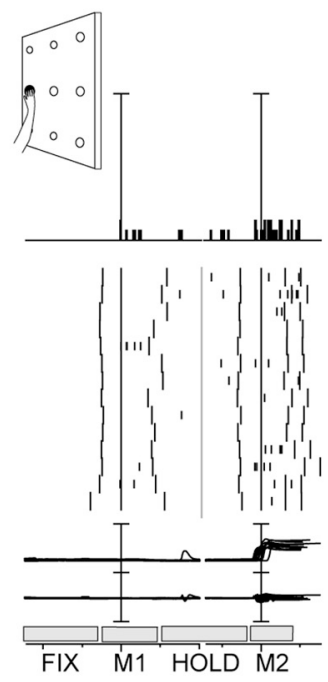

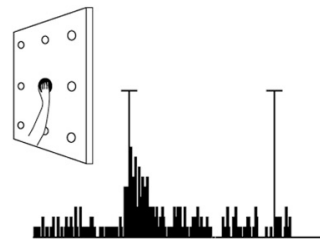
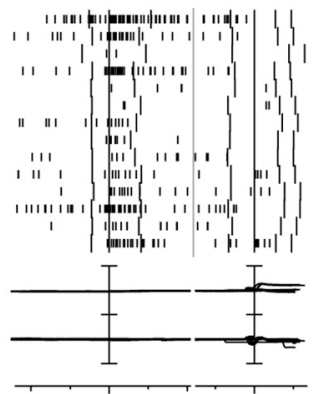

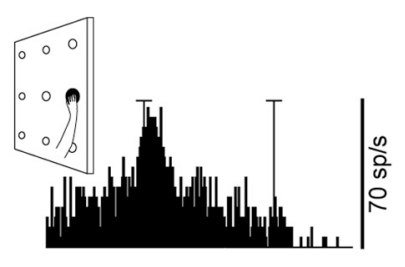

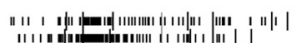

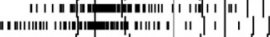

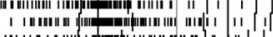
1

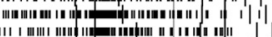

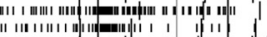
(11) 11

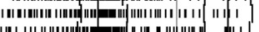

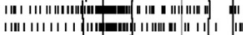

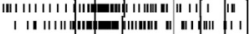
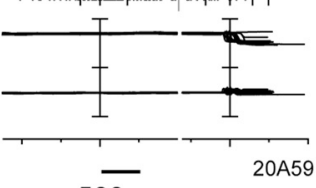

$500 \mathrm{~ms}$

FIGURE 5 | (A) Experimental set-up and time course of a frontal reaching task. Reaching movements were performed in darkness, from a home-button (black rectangle) toward one out of nine targets (open circles) located on a panel in front of the animal. (B) Incidence of V6A cells spatially modulated in the reaching task. Columns indicate the percentages of spatially modulated V6A cells during outward reaching movements (M1), and static position of the arm in the peripersonal space (HOLD). "Proper" activity = "raw" activity - FIX activity. (C) Example of spatially tuned modulation of reach-related activity. Neuron spatially tuned in M1, preferring rightward M1 movements. Neural activity and eye-traces have been aligned twice in each inset, with the onsets of outward (1st) and inward (2nd) reach movements. The mean duration of epochs FIX, M1, HOLD, M2 is indicated in the bottom left inset. Behavioral markers on rasters from left to right: LED color change, outward movement onset, outward movement end, LED offset, inward movement onset, inward movement end, end of trial. Bin size in peri-event time histograms: $15 \mathrm{~ms}$; eye traces: scale bar, $60^{\circ}$. Other details as in Figure 2. Modified from Fattori et al. (2005)
Kanai et al. (2010) observed that the perceptual switch rate between bistable structure-from-motion stimuli in humans was correlated with macroscopic anatomical features of an SPL area that mostly corresponded with the region identified by Yantis and colleagues.

Recent evidence suggests that SPL is also recruited in tasks involving working memory. Koenigs et al. (2009) found that humans with lesions in SPL displayed deficits in working memory tests that required the manipulation and rearrangement of working memory, but not the simple retrieval of it. The authors found that the manipulation in the working memory was impaired not only for visuospatial stimuli, but also for verbal-auditory ones. Furthermore, their findings are consistent with a meta-analysis of several neuroimaging studies that highlighted the involvement of SPL in the updating of the working memory (Wager and
Smith, 2003). Finally, activity in SPL has been linked with mental navigation (Ino et al., 2002) and visuomotor learning (Inoue et al., 2000). In summary, the above studies strongly suggest that beyond its role in goal directed motor behavior, the cortex of SPL is also involved in processing cognitive information to enable the perception of a continuously changing environment.

\section{CONCLUSION}

In this review we have presented evidence that the cortex of the SPL, in particular area V6A processes signals related to eye position in depth. We showed that these signals can be used in simple spatial computations like the encoding of the location of visual targets in $3 \mathrm{D}$ space, but also in more "cognitive" ones, like the encoding of peripersonal space in terms of reachability by the arm. In addition, we reviewed studies in humans that suggest the 
involvement of SPL in other cognitive processes. Overall, these findings rather than challenging the classic view that SPL play a role as an interface between sensory information and motor action, highlight the fact that this interface shows a remarkable flexibility and is influenced by attention, learning, and other cognitive factors.

\section{REFERENCES}

Ahissar, E., Vaadia, E., Ahissar, M., Bergman, H., Arieli, A., and Abeles, M. (1992). Dependence of cortical plasticity on correlated activity of single neurons and on behavioral context. Science 257, 1412-1415.

Akao, T., Kurkin, S. A., Fukushima, J., and Fukushima, K. (2005). Visual and vergence eye movement-related responses of pursuit neurons in the caudal frontal eye fields to motionin-depth stimuli. Exp. Brain Res. 164, 92-108.

Andersen, R. A., Bracewell, R. M., Barash, S., Gnadt, J. W., and Fogassi, L. (1990). Eye position effects on visual, memory, and saccade-related activity in areas LIP and 7a of macaque. J. Neurosci. 10, 1176-1196.

Andersen, R. A., and Cui, H. (2009). Intention, action planning, and decision making in parietalfrontal circuits. Neuron 63, 568-583.

Andersen, R. A., and Mountcastle, V. B. (1983). The influence of the angle of gaze upon the excitability of the light-sensitive neurons of the posterior parietal cortex. J. Neurosci. 3, 532-548.

Avillac, M., Deneve, S., Olivier, E., Pouget, A., and Duhamel, J. R. (2005). Reference frames for representing visual and tactile locations in parietal cortex. Nat. Neurosci. 8, 941-949.

Bakola, S., Gamberini, M., Passarelli, L., Fattori, P., and Galletti, C. (2010). Cortical connections of parietal field $\mathrm{PEc}$ in the macaque: linking vision and somatic sensation for the control of limb action. Cereb. Cortex 20, 2592-2604.

Barash, S., Bracewell, R. M., Fogassi, L., Gnadt, J. W., and Andersen, R. A. (1991). Saccade-related activity in the lateral intraparietal area. II. Spatial properties. J. Neurophysiol. 66, 1109-1124.

Battaglia-Mayer, A., and Caminiti, R. (2002). Optic ataxia as a result of the breakdown of the global tuning fields of parietal neurones. Brain 125, 225-237.

Battaglia-Mayer, A., Ferraina, S., Genovesio, A., Marconi, B.,
Squatrito, S., Molinari, M., Lacquaniti, F., and Caminiti, R. (2001). Eye-hand coordination during reaching. II. An analysis of the relationships between visuomanual signals in parietal cortex and parieto-frontal association projections. Cereb. Cortex 11, 528-544.

Battaglia-Mayer, A., Mascaro, M., Brunamonti, E., and Caminiti, R. (2005). The over-representation of contralateral space in parietal cortex: a positive image of directional motor components of neglect? Cereb. Cortex 15, 514-525.

Baylis, G. C., and Baylis, L. L. (2001). Visually misguided reaching in Balint's syndrome. Neuropsychologia 39, 865-875.

Bhattacharyya, R., Musallam, S., and Andersen, R. A. (2009). Parietal reach region encodes reach depth using retinal disparity and vergence angle signals. J. Neurophysiol. 102, 805-816.

Bremmer, F., Distler, C., and position effects in monkey cortex. II. pursuit- and fixation-related activity in posterior parietal areas LIP and 7A. J. Neurophysiol. 77, 962-977.

Breveglieri, R., Galletti, C., Gamberini, (2006). Somatosensory cells in area $\mathrm{PEc}$ of macaque posterior parietal cortex. J. Neurosci. 26, 3679-3684.

Breveglieri, R., Hadjidimitrakis, K., Bosco, A., Sabatini, S. P., Galletti, C., and Fattori, P. (2012). Eye position encoding in three-dimensional space: integration of version and vergence signals in the medial posterior parietal cortex. J. Neurosci. 32, 159-169.

Breveglieri, R., Kutz, D. F., Fattori, P., Gamberini, M., and Galletti, C. (2002). Somatosensory cells in the parieto-occipital area V6A of the macaque. Neuroreport 13, 2113-2116.

Buneo, C. A., Jarvis, M. R., Batista, A. P., and Andersen, R. A. (2002). Direct visuomotor transformations for reaching. Nature 416, 632-636.

Cavina-Pratesi, C., Monaco, S., Fattori, P., Galletti, C., McAdam, Hoffmann, K. P. (1997). Eye M., Passarelli, L., and Fattori, P.

\section{ACKNOWLEDGMENTS}

Funded by EU FP7-IST-217077-EYESHOTS, by Ministero dell'Universita e della Ricerca (Italy), and by Fondazione del Monte di Bologna e Ravenna (Italy). The authors wish to thank Dr. L. Passarelli, Dr. M. Gamberini, and F. Bertozzi for help with figures.

T. D., Quinlan, D. J., Goodale, M. A., and Culham, J. C. (2010) Functional magnetic resonance imaging reveals the neural substrates of arm transport and grip formation in reach-to-grasp actions in humans. J. Neurosci. 30, 10306-10323.

Chiu, Y. C., and Yantis, S. (2009). A domain-independent source of cognitive control for task sets: shifting spatial attention and switching categorization rules. J. Neurosci. 29, 3930-3938.

Cisek, P., and Kalaska, J. F. (2010) Neural mechanisms for interacting with a world full of action choices. Annu. Rev. Neurosci. 33 269-298.

Colby, C. L., and Duhamel, J. R. (1991). Heterogeneity of extrastriate visual areas and multiple parietal areas in the macaque monkey. Neuropsychologia 29 517-537.

Colby, C. L., Duhamel, J. R., and Goldberg, M. E. (1993). Ventral intraparietal area of the macaque: anatomic location and visual response properties. J. Neurophysiol. 69, 902-914.

Critchley, M. (1953). Tactile thought, with special reference to the blind. Brain 76, 19-35.

Culham, J. C., Cavina-Pratesi, C., and Singhal, A. (2006). The role of parietal cortex in visuomotor control: what have we learned from neuroimaging? Neuropsychologia 44, 2668-2684.

Culham, J. C., and Kanwisher, N. G. (2001). Neuroimaging of cognitive functions in human parietal cortex. Curr. Opin. Neurobiol. 11, 157-163.

Danckert, J., Goldberg, L., and Broderick, C. (2009). Damage to superior parietal cortex impairs pointing in the sagittal plane. Exp. Brain Res. 195, 183-191.

Dobbins, A. C., Jeo, R. M., Fiser, J., and Allman, J. M. (1998). Distance modulation of neural activity in the visual cortex. Science 281, 552-555.

Durand, J. B., Trotter, Y., and Celebrini, S. (2010). Privileged processing of the straight-ahead direction in primate area V1. Neuron 66, 126-137.
Esterman, M., Chiu, Y. C., TamberRosenau, B. J., and Yantis, S. (2009). Decoding cognitive control in human parietal cortex. Proc. Natl. Acad. Sci. U.S.A. 106, 17974-17979.

Fattori, P., Breveglieri, R., Marzocchi, N., Filippini, D., Bosco, A., and Galletti, C. (2009). Hand orientation during reach-to-grasp movements modulates neuronal activity in the medial posterior parietal area V6A. J. Neurosci. 29, 1928-1936.

Fattori, P., Kutz, D. F., Breveglieri, R., Marzocchi, N., and Galletti, C. (2005). Spatial tuning of reaching activity in the medial parietooccipital cortex (area V6A) of macaque monkey. Eur. J. Neurosci. 22, 956-972.

Fattori, P., Raos, V., Breveglieri, R., Bosco, A., Marzocchi, N., and Galletti, C. (2010). The dorsomedial pathway is not just for reaching: grasping neurons in the medial parieto-occipital cortex of the macaque monkey. J. Neurosci. 30, 342-349.

Ferraina, S., Brunamonti, E., Giusti, M. A., Costa, S., Genovesio, A., and Caminiti, R. (2009). Reaching in depth: hand position dominates over binocular eye position in the rostral superior parietal lobule. J. Neurosci. 29, 11461-11470.

Galletti, C., Battaglini, P. P., and Fattori, P. (1995). Eye position influence on the parieto-occipital area PO (V6) of the macaque monkey. Eur. J. Neurosci. 7, 2486-2501.

Galletti, C., Breveglieri, R., Lappe, M., Bosco, A., Ciavarro, M., and Fattori, P. (2010). Covert shift of attention modulates the ongoing neural activity in a reaching area of the macaque dorsomedial visual stream. PLoS ONE 5:e15078. doi: 10.1371/journal.pone. 0015078

Galletti, C., Fattori, P., Battaglini, P. P., Shipp, S., and Zeki, S. (1996). Functional demarcation of a border between areas V6 and V6A in the superior parietal gyrus of the macaque monkey. Eur. J. Neurosci. $8,30-52$.

Galletti, C., Fattori, P., Kutz, D. F., and Gamberini, M. (1999). Brain 
location and visual topography of cortical area V6A in the macaque monkey. Eur. J. Neurosci. 11, 575-582.

Galletti, C., Kutz, D., Gamberini, M., Breveglieri, R., and Fattori, P. (2003). Role of the medial parieto-occipital cortex in the control of reaching and grasping movements. Exp. Brain Res. 153, 158-170.

Gallivan, J. P., Cavina-Pratesi, C., and Culham, J. C. (2009). Is that within reach? fMRI reveals that the human superior parieto-occipital cortex encodes objects reachable by the hand. J. Neurosci. 29, 4381-4391.

Gallivan, J. P., McLean, A., and Culham, J. C. (2011). Neuroimaging reveals enhanced activation in a reachselective brain area for objects located within participants' typical hand workspaces. Neuropsychologia 49, 3710-3721.

Gamberini, M., Passarelli, L., Fattori, P., Zucchelli, M., Bakola, S., Luppino, G., and Galletti, C. (2009). Cortical connections of the visuomotor parietooccipital area V6Ad of the macaque monkey. J. Comp. Neurol. 513, 622-642.

Gamlin, P. D., and Yoon, K. (2000). An area for vergence eye movement in primate frontal cortex. Nature 407, 1003-1007.

Genovesio, A., and Ferraina, S. (2004). Integration of retinal disparity and fixation-distance related signals toward an egocentric coding of distance in the posterior parietal cortex of primates. J. Neurophysiol. 91, 2670-2684.

Godschalk, M., Mitz, A. R., van Duin, B., and van der Burg, H. (1995). Somatotopy of monkey premotor cortex examined with microstimulation. Neurosci. Res. 23, 269-279.

Goodale, M. A., and Milner, A. D. (1992). Separate visual pathways for perception and action. Trends Neurosci. 15, 20-25.

Gottlieb, J., and Snyder, L. H. (2010). Spatial and non-spatial functions of the parietal cortex. Curr. Opin. Neurobiol. 20, 731-740.

Graziano, M. S., and Cooke, D. F. (2006). Parieto-frontal interactions, personal space, and defensive behavior. Neuropsychologia 44, 2621-2635.

Graziano, M. S., Cooke, D. F., and Taylor, C. S. (2000). Coding the location of the arm by sight. Science 290, 1782-1786.

Hadjidimitrakis, K., Bertozzi, F., Breveglieri, R., Bosco, A., Dal Bo', G., Galletti, C., and Fattori,
P. (2011a). "Visually guided arm movements in 3D space: spatial tuning in fixation, preparation and reaching activity in monkey medial posterior parietal cortex," in Annual Meeting of the Society for Neuroscience, (Washington, DC).

Hadjidimitrakis, K., Breveglieri, R., Placenti, G., Bosco, A., Sabatini, S. P., and Fattori, P. (2011b). Fix your eyes in the space you could reach: neurons in the macaque medial parietal cortex prefer gaze positions in peripersonal space. PLoS ONE 6:e23335. doi: 10.1371/journal.pone. 0023335

Hayhoe, M. M., Shrivastava, A., Mruczek, R., and Pelz, J. B. (2003). Visual memory and motor planning in a natural task. J. Vis. 3, 49-63.

Heider, B., Karnik, A., Ramalingam, N., and Siegel, R. M. (2010). Neural representation during visually guided reaching in macaque posterior parietal cortex. J. Neurophysiol. 104, 3494-3507.

Husain, M., and Nachev, P. (2007). Space and the parietal cortex. Trends Cogn. Sci. 11, 30-36.

Ino, T., Inoue, Y., Kage, M., Hirose, S., Kimura, T., and Fukuyama, H. (2002). Mental navigation in humans is processed in the anterior bank of the parietooccipital sulcus. Neurosci. Lett. 322, 182-186.

Inoue, K., Kawashima, R., Satoh, K., Kinomura, S., Sugiura, M., Goto, R., Ito, M., and Fukuda, H. (2000). A PET study of visuomotor learning under optical rotation. Neuroimage $11,505-516$.

Inoue, Y., Takemura, A., Kawano, K., Kitama, T., and Miles, F. A. (1998). Dependence of short-latency ocular following and associated activity in the medial superior temporal area (MST) on ocular vergence. Exp. Brain Res. 121, 135-144.

Kanai, R., Bahrami, B., and Rees, G. (2010). Human parietal cortex structure predicts individual differences in perceptual rivalry. Curr. Biol. 20, 1626-1630.

Koenigs, M., Barbey, A. K., Postle, B. R., and Grafman, J. (2009). Superior parietal cortex is critical for the manipulation of information in working memory. J. Neurosci. 29, 14980-14986.

Lacquaniti, F., Guigon, E., Bianchi, L., Ferraina, S., and Caminiti, R. (1995). Representing spatial information for limb movement: role of area 5 in the monkey. Cereb. Cortex 5, 391-409.
Land, M. F., and Furneaux, S. (1997). The knowledge base of the oculomotor system. Philos. Trans. R. Soc. Lond. B Biol. Sci. 352, 1231-1239.

Land, M., Mennie, N., and Rusted, J. (1999). The roles of vision and eye movements in the control of activities of daily living. Perception 28, 1311-1328.

Law, I., Svarer, C., Rostrup, E., and Paulson, O. B. (1998). Parietooccipital cortex activation during self-generated eye movements in the dark. Brain 121( $\mathrm{Pt} 11)$ 2189-2200.

Macaluso, E., and Maravita, A. (2010). The representation of space near the body through touch and vision. Neuropsychologia 48 782-795.

Mascaro, M., Battaglia-Mayer, A., Nasi L., Amit, D. J., and Caminiti, R (2003). The eye and the hand: neural mechanisms and network models for oculomanual coordination in parietal cortex. Cereb. Cortex 13, 1276-1286.

Matelli, M., Govoni, P., Galletti, C., Kutz, D. F., and Luppino, G. (1998). Superior area 6 afferents from the superior parietal lobule in the macaque monkey. J. Comp. Neurol. 402, 327-352.

McGuire, L. M., and Sabes, P. N. (2011) Heterogeneous representations in the superior parietal lobule are common across reaches to visual and proprioceptive targets. J. Neurosci. 31, 6661-6673.

Nakamura, K., Chung, H. H., Graziano, M. S. A., and Gross, C. G. (1999). Dynamic representation of eye position in the parietooccipital sulcus. J. Neurophysiol. 81 , 2374-2385.

Pandya, D. N., and Seltzer, B. (1982) Intrinsic connections and architectonics of posterior parietal cortex in the rhesus monkey. J. Comp. Neurol. 204, 196-210.

Passarelli, L., Rosa, M. G., Gamberini, M., Bakola, S., Burman, K. J., Fattori, P., and Galletti, C. (2011) Cortical connections of area V6Av in the macaque: a visual-inpu node to the eye/hand coordination system. J. Neurosci. 31, 1790-1801.

Perenin, M. T., and Vighetto, A. (1988) Optic ataxia: a specific disruption in visuomotor mechanisms. I. different aspects of the deficit in reaching for objects. Brain 111(Pt 3), 643-674.

Pitzalis, S., Galletti, C., Huang, R. S., Patria, F., Committeri, G., Galati, G., Fattori, P., and Sereno, M. I. (2006). Wide-field retinotopy defines human cortical visual area V6. J. Neurosci. 26, 7962-7973.

Pouget, A., and Sejnowski, T. J. (1994). A neural model of the cortical representation of egocentric distance. Cereb. Cortex 4, 314-329.

Previc, F. H. (1998). The neuropsychology of 3-D space. Psychol. Bull. 124, 123-164.

Quinlan, D. J., and Culham, J. C. (2007). fMRI reveals a preference for near viewing in the human parieto-occipital cortex Neuroimage 36, 167-187.

Raos, V., Franchi, G., Galesse, V., and Fagassi, L. (2003). Somatotopic organization of the lateral part of area F2 (dorsal premotor cortex) of the macaque monkey. J. Neurophysiol. 89, 1503-1518.

Rosenbluth, D., and Allman, J. M. (2002). The effect of gaze angle and fixation distance on the responses of neurons in V1, V2, and V4. Neuron $33,143-149$.

Sakata, H., Shibutani, H., and Kawano, K. (1980). Spatial properties of visual fixation neurons in posterior parietal association cortex of the monkey. J. Neurophysiol. 43, 1654-1672.

Salinas, E., and Sejnowski, T. J. (2001). Gain modulation in the central nervous system: where behavior, neurophysiology, and computation meet. Neuroscientist 7 430-440.

Shipp, S., Blanton, M., and Zeki, S. (1998). A visuo-somatomotor pathway through superior parietal cortex in the macaque monkey: cortical connections of areas V6 and V6A. Eur. J. Neurosci. 10, 3171-3193.

Snyder, L. H., Batista, A. P., and Andersen, R. A. (1997). Coding of intention in the posterior parietal cortex. Nature 386, 167-170.

Viguier, A., Clement, G., and Trotter, Y. (2001). Distance perception within near visual space. Perception 30, $115-124$.

Wager, T. D., and Smith, E. E. (2003). Neuroimaging studies of working memory: a meta-analysis. Cogn. Affect. Behav. Neurosci. 3, 255-274.

Williams, A. L., and Smith, A. T (2010). Representation of eye position in the human parietal cortex. J. Neurophysiol. 104, 2169-2177.

Wolpert, D. M., Goodbody, S. J., and Husain, M. (1998). Maintaining internal representations: the role of the human superior parietal lobe. Nat. Neurosci. 1, 529-533. 
Yantis, S., Schwarzbach, J., Serences, J. T., Carlson, R. L., Steinmetz, M. A., Pekar, J. J., and Courtney, S. M. (2002). Transient neural activity in human parietal cortex during spatial attention shifts. Nat. Neurosci. 5, 995-1002.

Zipser, D., and Andersen, R. A. (1988). A back-propagation programmed network that simulates response properties of a subset of posterior parietal neurons. Nature 331, 679-684.

Conflict of Interest Statement: The authors declare that the research was conducted in the absence of any commercial or financial relationships that could be construed as a potential conflict of interest.
Received: 28 March 2012; accepted: 01 June 2012; published online: 19 June 2012.

Citation: Hadjidimitrakis K, Breveglieri $R$, Bosco A and Fattori P (2012) Threedimensional eye position signals shape both peripersonal space and arm movement activity in the medial posterior parietal cortex. Front. Integr. Neurosci. 6:37. doi: 10.3389/fnint.2012.00037
Copyright (C) 2012 Hadjidimitrakis, Breveglieri, Bosco and Fattori. This is an open-access article distributed under the terms of the Creative Commons Attribution Non Commercial License, which permits non-commercial use, distribution, and reproduction in other forums, provided the original authors and source are credited. 